\title{
A new wolf spider species of the genus Artoria from Western Australia (Araneae: Lycosidae)
}

\author{
Volker W. Framenau \\ Department of Terrestrial Zoology, Western Australian Museum, Locked Bay 49, \\ Welshpool D.C., Western Australia 6986, Australia \\ School of Animal Biology, University of Western Australia, Crawley, Western Australia 6009, Australia. \\ E-mail: volker.framenau@museum.wa.govau
}

\begin{abstract}
A new Australian wolf spider species, Artoria limmei, is described from south-westem Western Australia. Artorin now includes 24 species, of which 20 are found in Australia. Artoria limaci is common in suburban parks, reserves and gardens in and around Perth but also occurs further to the south. It appears to prefer moderately moist, low vegetation such as grassy areas among bushes and trees. The species is seasonal; adult males are active from late winter (July) into early summer (December) with a distinct peak in September, and females have been found from August to January peaking in November.
\end{abstract}

\section{INTRODUCTION}

The contribution of Carolus Linnaeus (17071778 ) to the naming of wolf spiders (family Lycosidae Sundevall 1833) was moderate. He described only four species, all originally in the genus Aranea Linnaeus 1758 (Platnick 2008): Lycosa tarantula (Linnaeus 1758) (the type species of Lycosa Latreille 1804) from the Mediterranean region; Pardosa palustris (Linnaeus 1758) from the Holarctic; and Aranea fumigata Linnaeus 1758 and Aranea saccata Linnaeus 1758, both today considered junior synomyms of Pardosa amentata (Clerck 1758) (Holarctic).

The genus Artoria Thorell 1877 currently includes 23 species from the Australasian and Pacific regions. Nineteen species have been described from Australia (one of which also occurs in New Caledonia and Vanuatu and one in Indonesia and the Philippines) (Framenau 2002, 2004, 2005; Framenau and Hebets 2007). Three species of Artoria are known from New Zealand (Vink 2002). Three further species from Africa are listed in the genus (Platnick 2008), but it is unlikely that these species belong in Artoria. Recent molecular studies suggest that Artoria as currently defined is not monophyletic, at least with respect to the New Zealand species (Murphy it al. 2006). Only a comprehensive phylogenetic analysis, including many of the more than 50 undescribed species of Artoria currently recognized in Australian collections, will be able to establish possible generic limits within this group of wolf spiders.
The aim of this study is to describe a new species of Artoria in honour of Carolus Linnaeus on the $250^{\text {th }}$ anniversary of the publication of the $10^{\text {th }}$ edition of his Systema Naturae. This species warrants description more than any other Western Australian Artoria, since it is one of the most common representatives of this genus in suburban Perth in addition to A. cingulipes Simon 1909 and A. flavimana Simon 1909.

\section{MATERIAL AND METHODS}

Descriptions are based on specimens preserved in $70 \%$ ethanol. Internal female genitalia were prepared for examination by submersion in $10 \%$ potassium hydroxide $(\mathrm{KOH})$ at room temperature for $10 \mathrm{~min}$. For clarity, the setae have been omitted from the illustrations of epigynes and male pedipalps. The morphological nomenclature follows Framenau $(2002,2005)$. All measurements are in millimeters $(\mathrm{mm})$.

Digital images were taken with a Leica DFC500 camera that was attached to a Leica MZ16A stereo microscope. Photographs were taken in different focal planes (ca. 40 images) and combined with the Leica Application Suite version 2.5.0R1.

\section{Abbreviations}

Measurements (adult spiders, if not otherwise stated): total length (TL), carapace length (CL) and width (CW), abdomen length (AL) and width (AW).

Eyes: anterior (AE), anterior median (AME), anterior lateral (ALE), posterior (PE), posterior 
median (PME), posterior lateral (PLE).

Collections: Queensland Museum, Brisbane (QM); Western Australian Museum, Perth (WAM).

\section{SYSTEMATICS}

\section{Family Lycosidae Sundevall 1833}

\section{Subfamily Artoriinae Framenau 2007}

\section{Artoria Thorell 1877}

\section{Type species}

Artoria parvula Thorell 1877 , by original designation.

\section{Artoria linnaei sp. nov.}

Figures $1 \mathrm{~A}-\mathrm{B}, 2 \mathrm{~A}-\mathrm{D}, 3$

\section{Material examined}

\section{Holotype}

Australia: Western Australia: 8 , Glenbourne

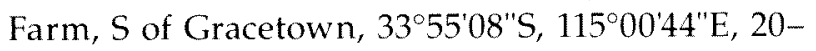
23 October 2000, dry pitfall trap, site 1, L. Marsh et al. (WAM T88459).

\section{Paratypes}

Australia: Western Australia: 1 o, 1 \%, Glen-

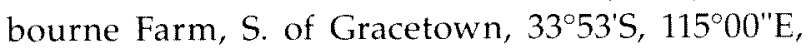
13-15 September 1997, dry pitfall trap, site 4, L. Marsh et al. (WAM T88460); 1 male, same data (WAM T88461).

\section{Other material examined}

Australia: Western Australia: 1 के, Attadale, $32^{\circ} 01^{\prime} \mathrm{S}, 115^{\circ} 48^{\prime} \mathrm{E}$ (WAM T55456); 1 ¿ , Bentley, $32^{\circ} 01^{\prime} \mathrm{S}, 115^{\circ} 55^{\prime} \mathrm{E}$ (WAM T65601); 1 \&, Bold Park, Perth, 31 56'29"S, 115'46'01"E (WAM T56406); 4 d, 3 ㅇ, same locality (WAM T68613); 2 ?, 1 q with spiderlings, same locality (WAM T68615); 2 १, same locality (WAM T68650); 1 \%, Bold Park, Perth, 31 ${ }^{\circ} 57^{\prime} 11^{\prime \prime} S, 1^{\circ} 45^{\prime} 50^{\prime \prime} \mathrm{E}$ (WAM T68616); 2 $\uparrow, 1$ q with spiderlings, same locality (WAM T68618); 17 o, 11 o, 1 juvenile, same locality (WAM T68636); 41 ठ, 16 \%, 1 juvenile, Bold Park, Perth, 31 56 $30^{\prime \prime} S, 115^{\circ} 46^{\prime} 27^{\prime \prime E}$ (WAM T68632); 1 o, 4 q, same locality (WAM T68614); 4 \%, Bold same locality (WAM T68617); 1 d, Bold Park,

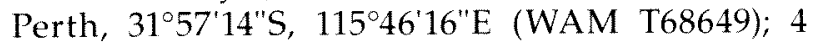
$\delta, 2$, same locality (WAM T68633); 3 o, 3 q, Brookdale, 32 $09^{\prime} 13^{\prime \prime} \mathrm{S}, 115^{\circ} 57^{\prime} 45^{\prime \prime} \mathrm{E}$ (WAM T84126); 1 o, same locality (WAM T84127); 1 o, Brookdale, $32^{\circ} 09^{\prime 2} 23^{\prime \prime}$, $115^{\circ} 57^{\prime} 43^{\prime \prime} \mathrm{E}$ (WAM T84125); 1 o, 1 ㅇ, Brookdale, 32 $09^{\prime} 17^{\prime \prime} \mathrm{S}, 115^{\circ} 57^{\prime} 46^{\prime \prime} \mathrm{E}$ (WAM T84128); 1 \&. Cannington Botanical Reserve, 32 01'29"S, $115^{\circ} 58^{\prime} 57^{\prime \prime} \mathrm{E}$ (WAM T55495); 1 \%, Canning Vale, Fairfield, $32^{\circ} 04^{\prime} \mathrm{S}, 115^{\circ} 54^{\prime} \mathrm{E}$ (WAM T55213); 1 \&. 30 miles E. of Collie, 33⒉'s, $116^{\circ} 55^{\prime} \mathrm{E}$ (WAM T56173); $1 \delta, 1 \&, 1$ juvenile, Coogee, $32^{\circ} 07^{\prime} \mathrm{S}, 115^{\circ} 46^{\prime} \mathrm{E}$
(WAM T55161); 1 o, 1 \&, Dianella, Cottonwood Crescent Bushland Reserve (labeled "Tuart Hill"), $31^{\circ} 52^{\prime} 49^{\prime \prime} \mathrm{S}, 1^{\circ} 5^{\circ} 51^{\prime} 30^{\prime \prime} \mathrm{E}$ (WAM T68674); 1 d, East Guildford, 28 Swan St, 31 $54^{\prime} \mathrm{S}, 115^{\circ} 59^{\prime} \mathrm{E}$ (WAM T56176); 1 \&, same locality (WAM T64086); 1 q, same locality (WAM T64606); 2 \&, same locality (WAM T64607); 80 , Glenbourne Farm, $S$ of Gracetown, $33^{\circ} 53^{\prime} \mathrm{S}, 115^{\circ} 00^{\prime} \mathrm{E}$ (WAM T88443); $3 \delta$, same locality (WAM T88444); 5 d, same locality (WAM T88445); 3 b, same locality (WAM T88447); 1 d, 1 \&, Glenbourne Farm, S of Gracetown,

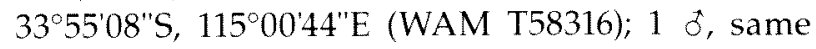
locality (WAM T88446); 1 b, Glenbourne Farm,

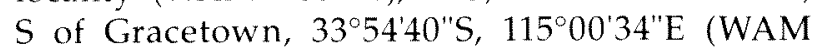
T88448); 1 t, Gnangara, $31^{\circ} 50^{\prime} \mathrm{S}, 115^{\circ} 50^{\prime} \mathrm{E}$ (WAM T55302); 5 o, 2 ㅇ, 1 juvenile, Guildford, $31^{\circ} 54^{\prime} \mathrm{S}$, $115^{\circ} 58^{\prime} \mathrm{E}$ (WAM T55567); 10 ठ, Haddleton Spring

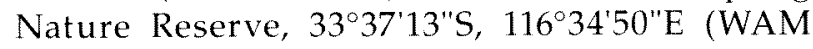
T55209); 1 'े. Hepburn Heights, $31^{\circ} 49^{\prime} 07^{\prime \prime} \mathrm{S}$, $115^{\circ} 46^{\prime} 11^{\prime \prime} \mathrm{E}$ (WAM T68577); 4 ơ, Hepburn Heights, 31 $49^{\circ} 02^{\prime \prime} S, 115^{\circ} 46^{\prime} 13^{\prime \prime} \mathrm{E}$ (WAM T68580); 3 \%, same locality (WAM T68587); 4 d, 1 q, Hepburn Heights, $31^{\circ} 49^{\prime} 06^{\prime \prime} S, 115^{\circ} 46^{\prime} 02^{\prime \prime} \mathrm{E}$ (WAM T68574); 2 \%, same locality (WAM T68579); 1 \%, 7 \% , Hepburn Heights, $31^{\circ} 48^{\prime} 57^{\prime \prime} S, 115^{\circ} 46^{\prime} 41^{\prime \prime}$ (WAM T68576); 52 d, 7 \%, same locality (WAM T68594); 1 \&, High

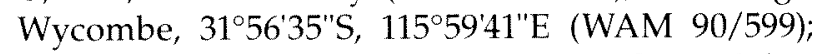
1 \%, same locality (WAM 90/600); 4 \%, Jandakot Airport, 32 05'31"S, 115 52'28"E (WAM 99/1-4); 9 5, 1 juvenile, same locality (WAM T68669); 4 d, 1 juvenile, same locality (WAM T68680); 9 o, 3 q, Jandakot Airport, 32 $05^{\prime} 36^{\prime \prime} \mathrm{S}, 115^{\circ} 52^{\prime} 39^{\prime \prime} \mathrm{E}$ (WAM T68646); 1 \%, same locality (WAM T68673); 1 o, 1 q, same locality (WAM T68679); 1 q, same locality (WAM T88522); 1 \%, Kings Park, Perth, 31 $57^{\prime}$ S, $115^{\circ} 50^{\prime} \mathrm{E}$ (WAM 78/211); 3 o, same locality (WAM 78/212-14); 1 o, Kings Park, Perth,

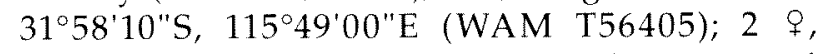
Kingsley, Montessori Primary School, Montessori Place, $31^{\circ} 48^{\prime} \mathrm{S}, 115^{\circ} 48^{\prime} \mathrm{E}$ (WAM T55242); 18 ठ, 11

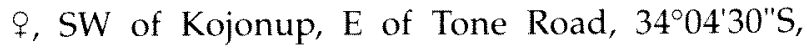
$116^{\circ} 51^{\prime} 49^{\prime \prime} \mathrm{E}$ (WAM T55210); 1 ?, Lake Clifton area, $32^{\circ} 47^{\prime} \mathrm{S}, 115^{\circ} 40^{\prime} \mathrm{E}$ (WAM T53826); 1 q, $\mathrm{N}$ of Lake

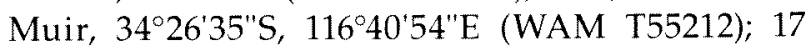
J. 4 q, 1 juvenile, Landsdale School, 31 $49^{\prime} 15^{\prime \prime}$, $115^{\circ} 51^{\prime} 01^{\prime \prime E}$ (WAM T68592); 1 o, Lesmurdie, Perth, $31^{\circ} 59^{\prime} \mathrm{S}, 116^{\circ} 02^{\prime} \mathrm{E}$ (WAM T64091); 4 , Marangaroo Reserve, 31 ${ }^{\circ} 49^{\prime} 38^{\prime \prime} \mathrm{S}, 115^{\circ} 50^{\prime} 04^{\prime \prime} \mathrm{E}$ (WAM T68584); 1 $\uparrow$, same locality (WAM T88472); 3 \&, Marangaroo

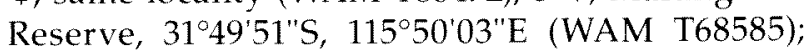
9 6., 1 \%, same locality (WAM T68589); 2 o, Mt Claremont, 31 $57^{\circ} 39^{\prime \prime} \mathrm{S}, 15^{\circ} 46^{\prime} 56^{\prime \prime} \mathrm{E}$ (WAM T68668); 1 \&. Mt Claremont, 31 $57^{\prime} 40^{\prime \prime}$ 'S, $115^{\circ} 46^{\prime} 00^{\prime \prime} \mathrm{E}$ (WAM T68667); 2 5े, 1 juvenile, same locality (WAM T68666); 4 ㅇ. Mt Henry, $32^{\circ} 01^{\prime} 58^{\prime \prime}$ S, $115^{\circ} 51^{\prime} 38^{\prime \prime} \mathrm{E}$ (WAM T68631); 1 d, same locality (WAM T68643); 7 \&, Mt Henry, 32 $01^{\prime} 53^{\prime \prime S}, 115^{\circ} 51^{\prime} 44^{\prime \prime E}$ (WAM T68638); 2 q, same locality (WAM T68647); 1 \%, Mt Lawley, Third Avenue, $31^{\circ} 56^{\prime} \mathrm{S}, 115^{\circ} 53^{\prime} \mathrm{E}$ (WAM 


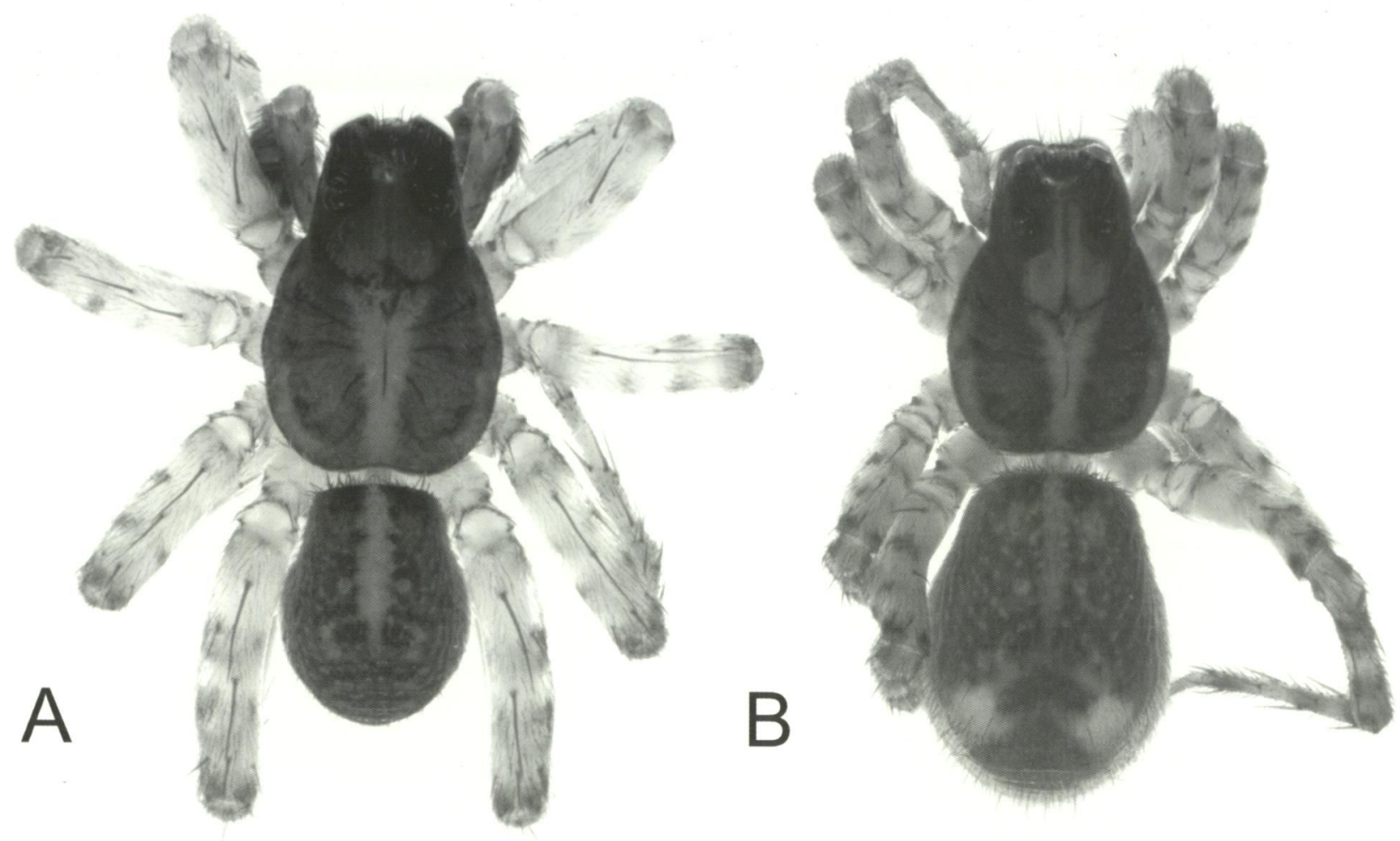

Figure 1 Artoria linnaei sp. nov. A, male holotype (WAM T88459); B, female paratype (WAM T88460), both from Glenbourne Farm, Western Australia. Body length: A, 3.27 mm; B, 4.50 mm.

T64062); 1 o, Mullaloo Beach, $6 \mathrm{~km}$ at $15 \mathrm{deg}$, $31^{\circ} 47^{\prime}$ S, $115^{\circ} 44^{\prime} \mathrm{E}$ (WAM T65611); 1 o, Nedlands, 7 Kingston St, 3157'56"S, 11548'46"E (WAM T53831); 1 \&, Parmelia, $32^{\circ} 14^{\prime} \mathrm{S}, 115^{\circ} 50^{\prime} \mathrm{E}$ (WAM T55241); 1 ㅇ, same locality (WAM T55425); 1 \%, same locality (WAM T65073); 10 ơ, 3 \%, 2 juveniles, Perth Airport, $31^{\circ} 58^{\prime} 34^{\prime \prime S}, 115^{\circ} 56^{\prime} 25^{\prime \prime E}$ (WAM T68662); 2 q, same locality (WAM T68663); 3 \%, 1 juvenile, same locality (WAM T68672); 13 के, 9 +, 1 juvenile, same locality (WAM T68675); 7 क, 5 ㅇ, Perth Airport, 31 $58^{\prime} 03^{\prime \prime} \mathrm{S}, 115^{\circ} 58^{\prime} 11^{\prime \prime E}$ (WAM T68653); 6 0, 10 क, 2 juveniles, same locality (WAM T68664); 1 ô, 4 क, 1 juvenile, same locality (WAM T68670); 1 q, same locality (WAM T68676); 1 \&, Perth Airport, $31^{\circ} 58^{\prime} 05^{\prime \prime S}, 115^{\circ} 58^{\prime} 05^{\prime \prime E}$ (WAM T56403); 5 \&, same locality (WAM T68657); 3 \$, 7 \%, same locality (WAM T68682); 3 , same locality (WAM T88484); 5 o, 2 ㅇ, Perth Airport, $31^{\circ} 58^{\prime} 36^{\prime \prime} \mathrm{S}, 115^{\circ} 58^{\prime} 28^{\prime \prime} \mathrm{E}$ (WAM 99/6-11); 36 o., 13 +10 juveniles, same locality (WAM T68656); 4 §, 2 , 1 juvenile, same locality (WAM T68658); 1 \&, Perth Airport, 31 $55^{\circ} 24^{\prime \prime}$, $115^{\circ} 58^{\prime} 40^{\prime \prime}$ (WAM T55491); 1 q, same locality (WAM T55503); 1 q, Perth Airport, 31 $55^{\circ} 25^{\prime \prime} S, 115^{\circ} 58^{\prime} 40^{\prime \prime E}$ (WAM T55504); 1 ô, Pinnaroo Valley Cemetery, 31\%47'52"S, 11546'43"E (WAM T64089); 4 ơ, Reabold Hill, $31^{\circ} 57^{\prime} \mathrm{S}, 115^{\circ} 46^{\prime} \mathrm{E}$ (WAM T42143); 1 \%, same locality (WAM T56174); 19 ô, 8 \%, same locality (QM S64096); 1 of, South Bunbury, Punchbowl Caravan

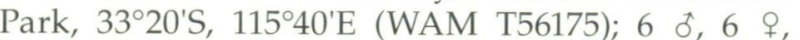

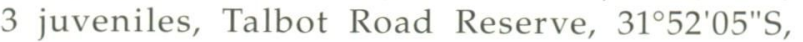

$116^{\circ} 03^{\prime} 04^{\prime \prime E ~(W A M ~ T 68583) ; ~} 24$ ơ, 3 ㅇ, 5 juveniles, same locality (WAM T68593); 3 ㅇ, same locality (WAM T68645); 3 o, 4 \&, same locality (WAM T68652); 13 o, 2 \%, same locality (WAM T68659); 9 o, 6 ㅇ, Talbot Road Reserve, 31 52'23"S, 116 02'46"E (WAM T68642); 1 ㅇ, same locality (WAM T68660);

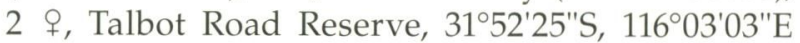
(WAM T68644); 3 o, 2 , 1 juvenile, Talbot Road Reserve, 31'52'24"S, 11602'52"E (WAM 99/12-17); 2 \&, same locality (WAM T68639); 4 ๙ึ, 1 ㅇ, 1 juvenile, same locality (WAM T68641); 15 0 0,7 , 3 juveniles, Trigg, 31 $52^{\prime} 9^{\prime \prime S}, 115^{\circ} 45^{\prime} 38^{\prime \prime E}$ (WAM T68588); 27 ㅇ, same locality (WAM T68586); 2 ㅇ, Trigg, 31 $52^{\prime} 45^{\prime \prime}$ S, $115^{\circ} 45^{\prime} 17^{\prime \prime E ~(W A M ~ T 68575) ; ~} 15$ , 1 juvenile, same locality (WAM T68582); 2 \$, 2 क, Trigg, 315' $30^{\prime \prime S}, 115^{\circ} 45^{\prime} 35^{\prime \prime} \mathrm{E}$ (WAM T68590); 2

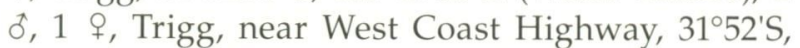
11545'E (WAM 99/174-6); 1 , same locality (WAM T68683); 1 क, same locality (WAM T68678); 1 q, Tuart Hill, 3152'50"S, 11551'32"E (WAM T68665); 22 §, 5 \%, 2 juveniles, same locality (WAM T68619);

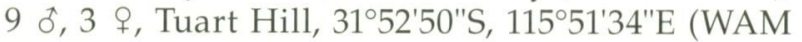
T68671); 1 to, same locality (WAM T68677); 2 , Tuart Hill, 3152'50"S, 11551'34"E (WAM 99/1819); 1 ㅇ, University of Western Australia Shenton Park Field Station, $31^{\circ} 57^{\prime} 02^{\prime \prime}$, $115^{\circ} 48^{\prime} 05^{\prime \prime}$ E (WAM T51415); 1 q, Warwick Open Space, 31 50'33"S, 11549'00"E (WAM T68581); 20 6, 5 क, Woodman Point, $32^{\circ} 07^{\prime} 58^{\prime \prime} S, 115^{\circ} 45^{\prime} 29^{\prime \prime E}$ (WAM T68635); 2 ㅇ, 4 juveniles, same locality (WAM T68640); 21

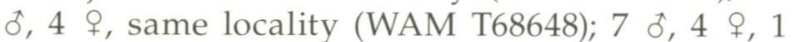




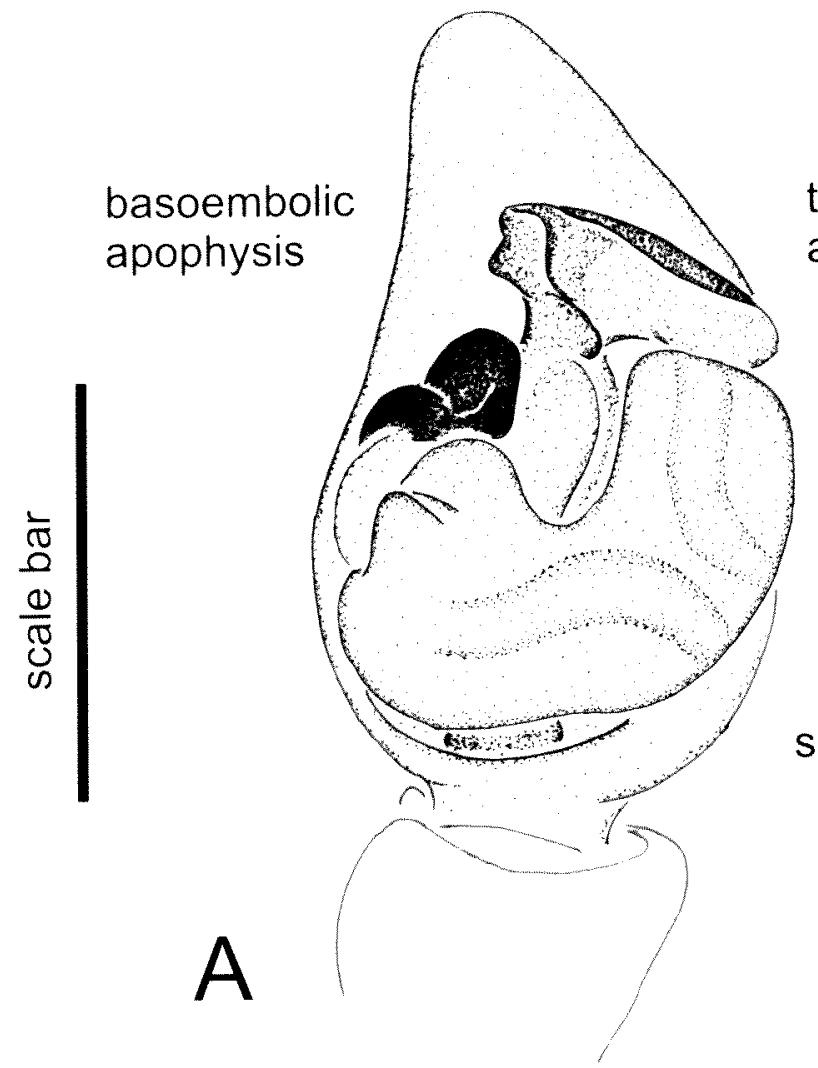

scape-like process

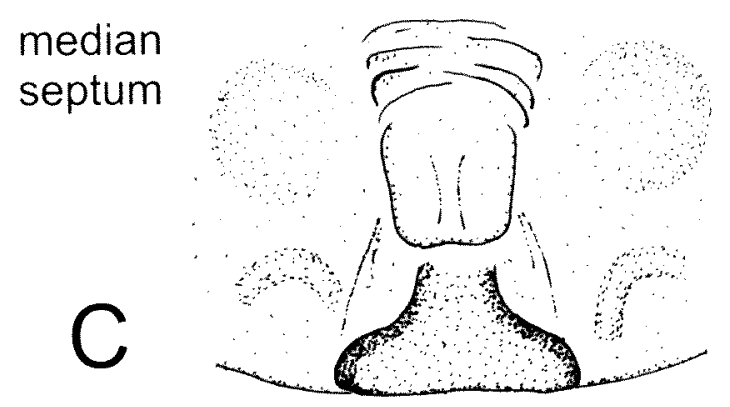

tegular apophysis

subtegulum

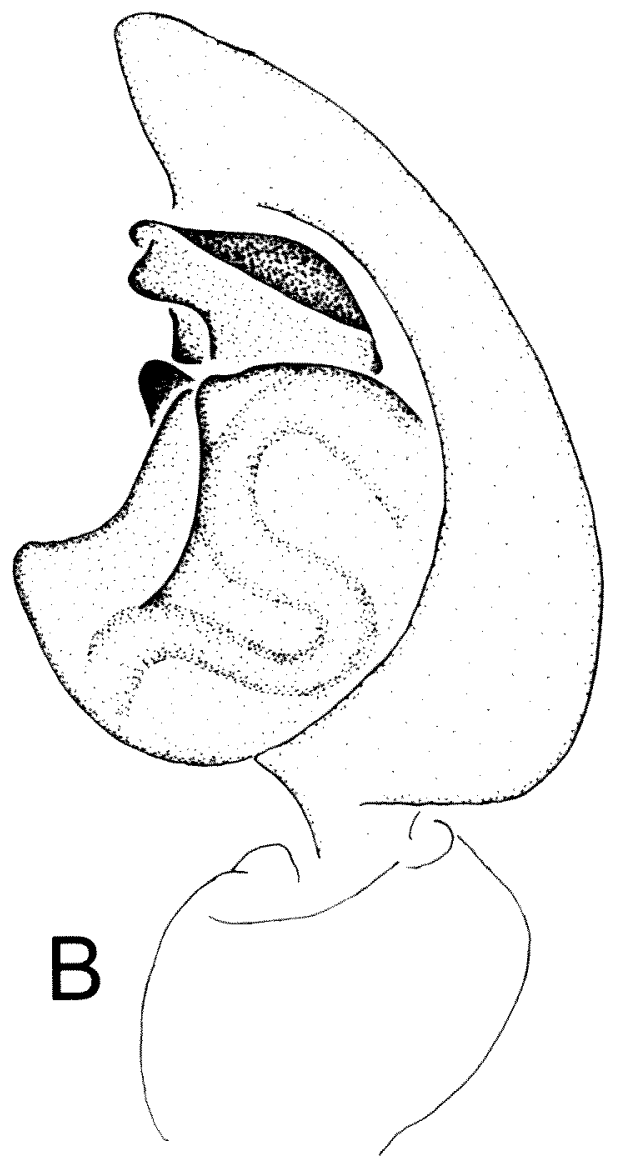

\section{spermathecal}

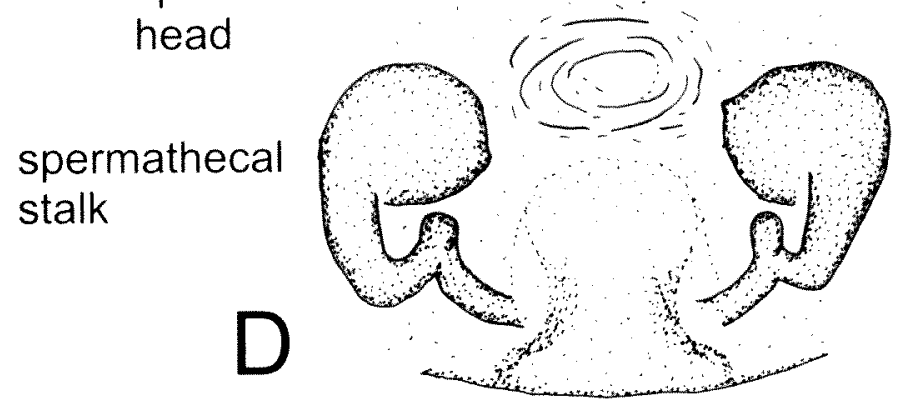

Figure 2 Artoria linnaei sp. nov., male paratype (WAM T88461, from Glenbourne Farm, Western Australia): A, left pedipalp, ventral view; $B$, left pedipalp, retrolateral view. Female paratype (WAM T88460, from Glenbourne Farm, Western Australia): C, epigyne, ventral view; D, epigyne, dorsal view. Scale bars: $A, B=0.37 \mathrm{~mm} ; C, D=$ $0.25 \mathrm{~mm}$.

juvenile, same locality (WAM T68654); 5 , same locality (WAM T68655); 23 0, 2 ?, Woodman Point, $32^{\circ} 07^{\prime} 50^{\prime \prime} \mathrm{S}, 115^{\circ} 45^{\prime} 26^{\prime \prime}$ (WAM T68661); 3 \& , 2 juveniles, same locality (WAM T88482); 3 \& 1 \&, 2 juveniles, Woodman Point, $32^{\circ} 07^{\prime} 50^{\prime \prime} \mathrm{S}, 115^{\circ} 45^{\prime} 28^{\prime \prime} \mathrm{E}$ (WAM 99/20-25); 6 \%, Woodman Point, $32^{\circ} 07^{\prime} 47^{\prime \prime} S$, $115^{\circ} 45^{\prime} 23^{\prime \prime} \mathrm{E}$ (WAM T68634); 7 \&, 3 \&, 1 juvenile, same locality (WAM T68637); 16 o, 3 +, 3 juveniles, same locality (WAM T68651); 10 , Wungong Dam, $32^{\circ} 11^{\prime} 38^{\prime \prime} \mathrm{S}, 116^{\circ} 03^{\prime} 36^{\prime \prime} \mathrm{E}$ (WAM T53793); 1 i, same locality (WAM T53794); 1 , same locality (WAM T53821); 4 oै, 1 ९, same locality (WAM T53822).

\section{Diagnosis}

Males of Artoria linnaei differ from all other species in Artoria by a unique ventral protrusion of the tegulum (Figure 2B) and the distinct, asymmetrically $\mathrm{T}$-shaped tegular apophysis (Figure 2A). The female genitalia are unique among species of Artoria, since the epigyne has a scape-like process anteriorly in combination with an inverted T-shaped median septum (Figure 2C).

\section{Description}

Male (based on holotype) 
Carapace (Figure 1A): brown; light brown median band that is darker and as wide as PLE anteriorly but gradually narrowing towards posterior margin; indistinct light brown submarginal bands; dark grey radial pattern; carapace covered with few silvery setae, denser in median and submarginal bands and in cephalic region; brown bristles between lateral eyes and, in pairs, between posterior median and anterior median eyes; two long bristles below anterior lateral eyes. Eyes: anterior row of eves strongly procurved, narrower than posterior row of eyes. Sternmm: yellow brown, darker centrally and marginally; brown bristles mainly towards margins. Labium: brown; frontal rim white. Chelicerae: glabrous light brown with grey pigmentation; few black setae medially; three retromarginal teeth, with the basal shortest; three promarginal teeth, with the middle largest. Pedipalp (Figures 2A-B): cymbium with short, dense setae dorso-apically; tegular apophysis asymmetrically $T$-shaped and with ventrally bent apical edge; tegulum with distinct ventral protrusion (Figure 1B). Abdomen (Figure 1A): mottled olive brown-olive grey, with distinct yellow-brown lanceolate cardiac mark; covered with silvery-white setae, which are particularly dense in cardiac mark, and few black setae; venter yellow-brown; covered mainly with whitish and few brown setae; spinnerets vellow-brown. Legs: leg formula IV $>$ III $>$ I $>$ II; light brown, with indistinct darker annulations. Spination of leg $\mathrm{l}$ : femur with 3 dorsal and 1 apicoprolateral; tibia with 2 ventral pairs and 2 prolateral; metatarsus with 3 ventral pairs.

\section{Female (based on paratype)}

Carapace (Figure 1B): as for male, median band lighter anteriorly and reaching between PME. Sternum as for male, marginally darker. Labium, Chelicerae: as for male. Epigyne (Figures 2C-D): with inverted T-shaped median septum and scapelike anterior process in ventral view (Figure 2C); spermathecal heads large, spherical in dorsal view (Figure 2C), with spermathecal stalks distinctly kinked midway. Abtomen (Figure 1B): as for male, but generally lighter and with two large light brown spots in posterior half; venter light brown with irregular grey patches; all spinnerets vellowbrown. Legs: leg formula IV > III > I > II; light brown, distinct dark annulations. Spination of leg 1: femur with 3 dorsal, 1 apicoprolateral; tibia with 2 ventral pairs and 2 prolateral; metatarsus with 3 ventral pairs and 1 apicoventral.

\section{Measurements}

Male holotype (female paratype): TL 3.27 (4.50) CL 1.93 (2.16), CW 1.27 (1.46). Eves: AME 0.07 (0.06), ALE $0.05(0.05)$, PME $0.25(0.23)$, PLE 0.15
(0.17). Row of eyes: AE $0.36(0.40)$, PME $0.65(0.67)$, PLE $0.70(0.77)$. Sternum (length/width) $0.89 / 0.69$ (0.92/0.77). Labium (length/width) $0.27 / 0.25$ (0.29/0.27). AL 1.35 (2.39), AW 1.00 (1.69). Legs: Lengths of segments (femur + patella/tibia + metatarsus + tarsus $=$ total length): Pedipalp 0.62 $+0.50+-0.65=1.77,11.19+1.58+0.92+0.54=$ $4.24,111.12+1.39+1.00+0.50=4.00,1111.19+1.39$ $+1.23+0.54=4.35$, IV $1.42+1.89+1.85+0.69=$ 5.85 (Pedipalp $0.69+0.66+-+0.42=1.77,11.16+$ $1.50+0.92+0.54=4.12,111.08+1.39+0.89+0.50$ $=3.85,1111.16+1.31+1.12+0.46=4.04$, IV $1.39+$ $1.85+1.73+0.65=5.62)$.

\section{Variation}

Males (females) (range, mean \pm s.d.): TL 3.14-3.57, $3.35 \pm 0.14 ; n=13 ; C L 1.76-2.09,1.92 \pm 0.09 ; n=13$; CW 1.24-1.48, $1.35 \pm 0.07 ; \mathrm{n}=13$ (TL 3.33-5.05, 4.24 $\pm 0.60, \mathrm{n}=12 ; \mathrm{CL} 1.76-2.52,2.09 \pm 0.27, \mathrm{n}=12 ; \mathrm{CW}$ $1.29-1.86,1.53 \pm 0.21 ; n=12)$.

The light patches in the posterior half of the abdomen as described for the paratype female above are discolourations that are quite common in many species of Artoria, in particular females, but are not present in all specimens.

\section{Remarks}

The presence of a basoembolic apophysis on the male pedipalp (Figure 2A) clearly identifies the new species as a member of the wolf spider subfamily Artoriinae (see Framenau 2007). Here it most closely conforms to the generic description of Artoria based on the shape of the basoembolic and tegular apophyses (Framenau 2002, 2005). However, it is possible that the unique genitalic features of Artoria limnat, in particular the tegular protrusion and the epigynal scape-like process, in combination with the unusual leg formula (third instead of first leg being the second longest) may warrant the establishment of a new genus pending a comprehensive phylogenetic revision of Artoria.

\section{Life history and habitat preferences}

Artoria limmei is commonly found in suburban parks, reserves and gardens in metropolitan Perth. It appears to prefer moderately moist, low vegetation such as grassy areas under trees and bushes. The species is seasonal; adult males are active from late winter (July) into early summer (December) with a distinct peak in september, and females have been found from August to January peaking into November. This phenology may be responsible for the limited records south of Perth as collections are rarely made in winter. Only two females carrying spiderlings have been found in Perth (Bold Park) in a pitfall trapping period spanning from September to November. 


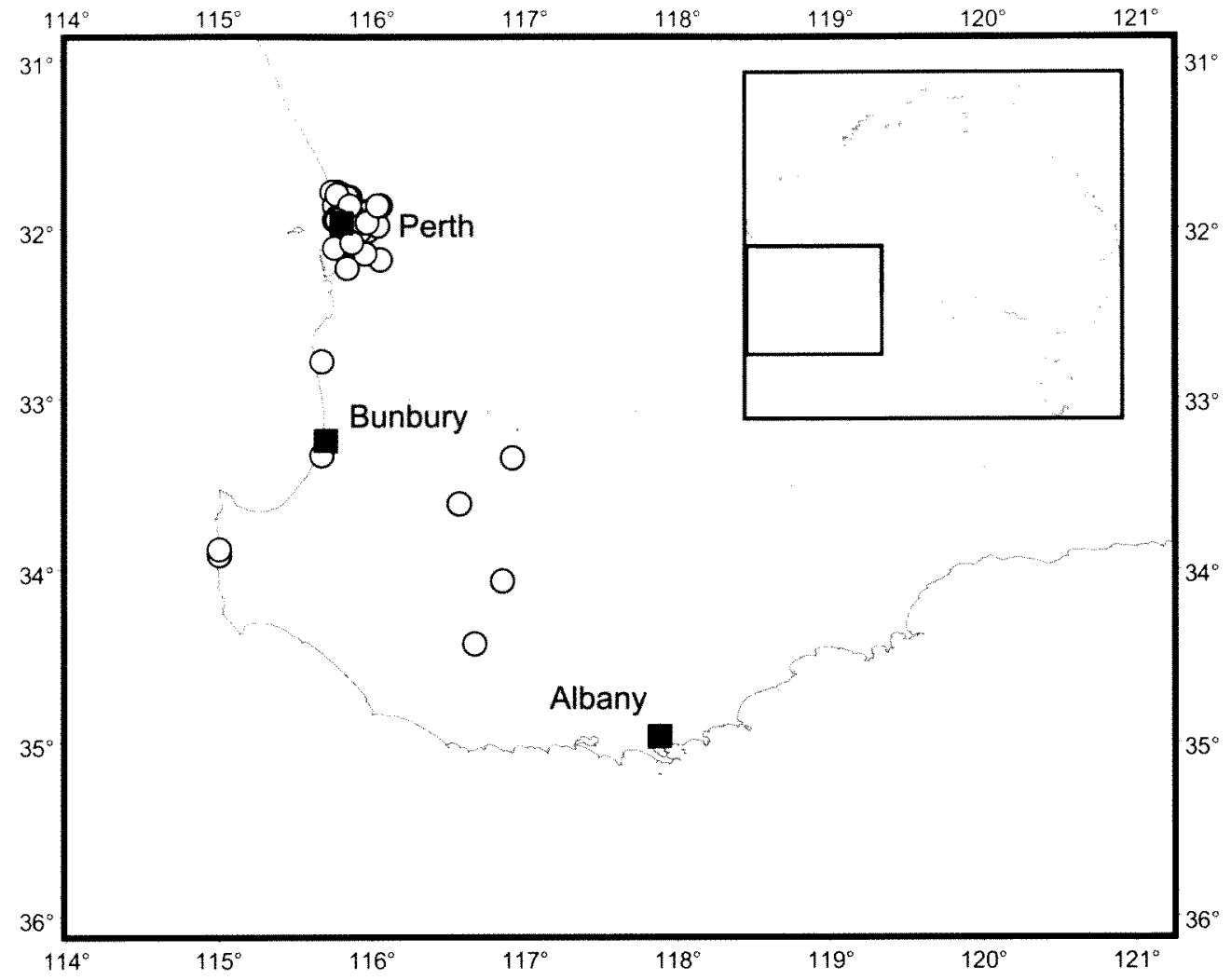

Figure 3 Distribution records of Artoria linnaci in Western Australia.

\section{Distribution}

Artoria linnaei was found in southwest Western Australia, most commonly in and around Perth (Figure 3).

\section{ACKNOWLEDGEMENTS}

I am thankful to Mark Harvey and Paul Doughty (Western Australian Museum) for the invitation to contribute to this special volume of the Records of the Western Australian Museum. The Australian Biological Resources Study (ABRS) provided funding to myself and Nikolaj Scharff (Zoological Museum, University of Copenhagen) for a revision of the Australian orb-web spiders of the subfamily Araneinae whilst this manuscript was written up (grant no: 205-24). ABRS also provided initial funding (2002-2005) to Mark Harvey and Andy Austin (University of Adelaide) for a revision of the wolf spiders of Australia.

\section{REFERENCES}

Framenau, V.W. (2002). Review of the wolf spider genus Artoria Thorell (Araneae, Lycosidae). Invertebrate Systematics 16: 209-235.

Framenau, V.W. (2004) [imprint date 2003]. Two alpine wolf spiders of Australia: Artoria alta sp. nov., and the male of Lycosa musgravei McKay, 1974 (Araneae, Lycosidae). Procedings of the Royal Soctety of Victoria 115: $27-34$.
Framenau, V.W. (2005). The wolf spider genus Artoria in Australia: new synonymies and generic transfers (Araneae, Lycosidae). Records of the Western Australian Musetm 22: 265-292.

Framenau, V.W. (2007). Revision of the new Australian genus Artoriopsis in a new subfamily of wolf spiders, Artoriinae (Araneae: Lycosidae). Zootaxa 1391: 1-34.

Framenau, V.W. and Hebets, E. A. (2007). A review of leg ornamentation in male wolf spiders, with the description of a new species from Australia, Artoria schizocoides (Araneae, Lycosidae). Journal of Arachnology 35: 89-101.

Murphy, N.P., Framenau, V.W., Donellan, S.C., Harvey, M.S., Park, Y.-C. and Austin, A.D. (2006). Phylogenetic reconstruction of the wolf spiders (Araneae: Lycosidae) using sequences from the $12 S$ rRNA, $28 \mathrm{~S}$ rRNA, and NADH1 genes: implications for classification, biogeography, and the evolution of web building behaviour. Molecular Phylogenetics and Evolution 38: 583-602.

Platnick, N.I. (2008). The World Spider Catalog, Version 8.5 http://research.amnh.org/entomology/spiders/ catalog/INTRO1.html. American Museum of Natural History (verified 8 April 2008)

Vink, C.J. (2002). Lycosidae (Arachnida: Araneae). Fauna of New Zealand 44: 1-94.

Manuscript received 9 April 2008; accepted 13 May 2008. 